\title{
A relevant Subspace based Contextual Outlier Mining Algorithm
}

\author{
Jifu Zhang ${ }^{\mathrm{a}}$, Xiaolong $\mathrm{Yu}^{\mathrm{a}}$, Yonghong $\mathrm{Li}^{\mathrm{a}}$, Sulan Zhang ${ }^{\mathrm{a}}$, Yaling Xun ${ }^{\mathrm{a}}$, and Xiao Qin ${ }^{\mathrm{b}}$ \\ ${ }^{a}$ School of Computer Science and Technology, Taiyuan University of Science and Technology, Taiyuan, P. R. China 030024 \\ ${ }^{\mathrm{b}}$ Department of Computer Science and Software Engineering, Auburn University, Auburn, AL, USA 36849-5347
}

E-mail: jifuzh@sina.com

\begin{abstract}
:
For high-dimensional and massive data sets, a relevant subspace based contextual outlier detection algorithm is proposed. Firstly, the relevant subspace, which can effectively describe the local distribution of the various data sets, is redefined by using local sparseness of attribute dimensions. Secondly, a local outlier factor calculation formula in the relevant subspace is defined with probability density of local data sets, and the formula can effectively reflect the outlier degree of data object that does not obey the distribution of the local data set in the relevant subspace. Thirdly, attribute dimensions of constituting the relevant subspace and local outlier factor are defined as the contextual information, which can improve the interpretability and comprehensibility of outlier. Fourthly, the selection of $\mathrm{N}$ data objects with the greatest local outlier factor value is defined as contextual outliers. In the end, experimental results validate the effectiveness of the algorithm by using UCI data sets.
\end{abstract}

\section{Keywords:}

Contextual outlier; Relevant subspace; Interpretability and comprehensibility; Local sparsity; Probability density.

\section{INTRODUCTION}

Outlier is a general pattern or behavior, which significantly deviates from the other data. This type of data are inconsistent with other existing data[1], and often contain a large number of information which is not easily to be found but offers a very high level of research and application value. Outlier mining, an important branch of data mining and data analysis, has been widely used in astronomical spectroscopy[2], credit card fraud[3], network intrusion mining[4,5], data cleaning[6] and so on. At present, outlier mining algorithms can be broadly classified into such methods as statistics-based [7], distance-based[8], densitybased[9,10], deviation-based [11], angle-based methods [3, 12] and so on. However these methods assume that each dimension plays a uniform contribution for detecting outliers [13]. The existence of some irrelevant dimensions will increase effects of "disaster dimension" on mining results, and lead to unable to find some outliers hidden in the subspace.

For the high-dimensional and massive data, the effectiveness and efficiency of outlier mining have been seriously influenced and some outliers hidden in the subspace may not be found. In most cases, the outliers are such data objects which are apparently inconsistent with the distribution features of the local dataset. But the inconsistent valuable information can be given in some attribute dimensions, while nothing important information is afforded on the other attribute dimensions and these dimensions are nearly irrelevant to detect the outliers[14]. Therefore, it can effectively reduce the disturbance of "disaster dimension" and find the hidden outliers by searching and deleting the attribute dimensions that cannot provide some valuable information in the high dimensional datasets. Aiming at the problem of highdimensional and massive data, a new contextual outlier mining algorithm based on relevant subspace is proposed in this paper. From the perspective of the local sparsity, the relevant subspace is redefined by making use of the local sparsity factor in the local dataset, and thus effectively described the distribution characteristics of all the local datasets. For the data objects in the relevant subspace, the calculation formula of the local outlier factor is given by using the probability density and Gaussian error function of the local dataset. Then the data attributes constituting the relevant subspace are regarded as the data objects' contextual information, and a contextual outlier mining algorithm is proposed, and accordingly it effectively reflects the inconsistency degree of the distribution in the local dataset and data objects in the relevant subspace, and provides the origin, meaning and features of outliers. The selection of $n$ data objects which are of the most outlier, are identified as the contextual outlier. Finally, the interpretability and performance of the algorithms are validated by taking UCI data as experimental datasets. In this paper, the main highlights and contributions are summarized as follows:

- The relevant subspace, which can effectively describe the local distribution of the various data sets, is redefined by using the local sparseness of attribute dimensions.

- We proposed that the attribute dimensions and outlier factor of data object in the relevant subspace are regarded as the contextual information, which can effectively explain the outlier's meaning and cause, and is benefit for the understanding of outlier.

- The computing formula of outlier factor in data object' relevant subspace is presented to define the object's outlier degree.

\section{RELATED WORK AND ANALYSIS}

Since mostly traditional outlier mining methods [7,8,9,10,11,12], detect outliers from all the dimensions of the data space, some irrelevant dimensions would have an effect on the efficiency and precision of outlier mining. It is one of the hot spots of highdimensional outlier mining to project the data onto a subspace for outliers mining $[14,15,16]$, however, it is more difficult to search for the meaningful subspace [15], and has the dimensionexponential complexity [14]. Aiming at outlier mining tasks, the typical methods of selecting meaningful subspace in the highdimensional data set mainly include two categories: one is sparsity subspace method[2,17,18] and the other is relevant subspace method $[13,19,20]$. The sparse subspace method projects all overall the data onto the sparse subspace according to sparse coefficient threshold given by the user, and the data objects which are contained in the subspace are determined as outliers. Therefore, the sparse subspace method is a type of global subspace method. Typical work are: c c.Agarwal et al used genetic algorithm to search for sparse subspace [17], but the algorithm performance 
was affected by the initial population, the completeness and accuracy of outlier mining's results cannot be guaranteed. Aiming at the deficiencies in [17], zhang Jifu et al, took concept lattice as a description tool for the subspace[2,18], and determined the sparse subspace through introducing the density coefficient. The method further improves the accuracy and completeness of the mining results, and has obtained better application in the astronomical spectrum data. However, the mining efficiency is lower because the construction of concept lattice is complex.

The basic idea of the relevant subspace method[13,14,16,19,20] is to look for the relevant subspace composed of some meaningful attribute dimensions in the datasets, from which the outliers are determined. The mainly used method is based on linear correlation of local reference datasets[13,19,20], as well as statistical models of local reference datasets[14, 16] and so on. Typical work includes: H.-P.Kriegel et al [19] proposed an outlier detection method in axis-parallel subspaces of high dimensional data (SOD). By sharing the nearest neighbor (SNN), the method looks for a similar subset for each data object, then determines the axisparallel linear correlation subspace in the similar subset based on the theory of attribute-based dimensions with low variance in the relevant subspace[19]. In the SOD, since the outlier degree of data objects is depicted with only one relevant subspace, this method will not be able to distinguish their outlier degree when the data objects are located in two or more than two subspace [14]. Clearly, it has some obvious deficiencies to detect outliers in the relevant subspace by using the mean-dimension of the Euclidean distance method. E.Muller et al[14] proposed a method to select the meaningful subspace from datasets by using KolmogorovSmirnov test statistics method, and search for non-uniform distribution subspace (relevant subspace)from low-to highdimensional in recursive ways, then regard the multiplicative of local outlier in each relevant subspace of data objects, as the ultimate outlier degree of the data objects, and accordingly solved the problem of outlier coefficient comparability in different subspaces. However, the complexity of determining all the relevant subspace in this algorithm is exponential time of the dimension [3], and the efficiency is low. Furthermore, the scalability of dimension for high dimensional datasets is poor, and unable to be adapted to the high-dimensional and massive data. F.Keller et al[16] using Monte Carlo methods to find relevant subspace set, and this method measures the outlier degree of objects from the local subset which the data objects correspond to each relevant subspace, but the relevant subspace are actually achieved from the point of global view. Hans-Peter Kriegel et al [13], on the basis of the theory of principal component analysis, detected outlier by using of Mahalanobis distance with gamma distribution methods. Relevant subspace achieved by this method is arbitrary subspace of linear correlation, and the relevant subspace has good adaptability to linear data. However, this method is based on the deviation from the local data distribution, and it is liable to make mistakes in the relevant subspace obtained from maximum likelihood estimation when outliers was in the regional which not obviously reflects of correlation. Therefore, the method needs enough local data to reflect the obviously deviation trend, and the complexity of this algorithm is the cube of the dimension and difficult to fit for the high dimensional data. In addition, Mohamed et al [20] find the relevant subspace by using of sparse density matrix, and make clustering analysis in the relevant subspace.
Most of outlier mining algorithms only to focus on the mining methods, and the reasons with the interpretation of outlier's generation are relatively few. So, the origins, meaning and characteristics of the outlier's generation have not been able to effectively explained. Guanting Tang et al proposed an outlier model with contextual information in [21]. On this basis, an outlier mining algorithms with the contextual information is presented, but the mining efficiency is low because the sphere of searching outlier is first narrowed, then outlier is searched with enumerating method. Wang and Davidson proposed a probabilistic approach based on random walks graph, which is essentially a homogeneous Markov chain as characterized by a transition matrix[22]. The principal eigenvector of the transition matrix gives the stationary distribution of the nodes being visited in the graph under a global random walk, so the method can simultaneously explore meaningful contexts and score contextual outliers.

\section{RELEVANT SUBSPACE AND LOCAL OUTLIER PROBABILITY}

According to the mining tasks on the different data sets, the data objects in the datasets only reflect the valuable information in some specific attribute dimensions, while there may little or no valuable information in other attribute dimensions[14]. Suppose DS be an arbitrary d-dimensional dataset, the attribute set $\mathrm{F}$, $F S=\left\{A_{1}, A_{2}, \ldots, A_{d}\right\}$, and $x_{i j}(i=1,2, \ldots n, j=1,2, \ldots d)$ denote the $j$-th attribute value of the $\mathrm{i}$-th data object. Referring to the reference [14] and [23], the basic concepts of the relevant subspace and local outlier probability are described as follows:

For an arbitrary subspace $S, S \subseteq F S$, data object $o, o \in D S$, and $\mathrm{N}(\mathrm{o}, \mathrm{S})$ is the nearest neighbor of $\mathrm{o}$. If $\mathrm{S}$ is a relevant subspace, then $\mathrm{N}(\mathrm{o}, \mathrm{S})$ is non-uniform distribution. If $\mathrm{S}$ is not a relevant subspace, then $\mathrm{N}(\mathrm{o}, \mathrm{S})$ is uniform distribution.

In [14], the non-uniform distribution of the subspace can effectively embody the valuable information of "outlier", while the uniform distribution of attribute dimensions cannot reflect the valuable information of "outlier". Therefore, the measurement and searching for the relevant subspace has become the key to detect outliers since the relevant subspace can effectively reflect the valuable information of "outlier".

In [23], for an arbitrary data object $o, o \in D S$, suppose $S$ be the correlation dataset, $S \subseteq \mathrm{DS}$ and $\mathrm{o} \in \mathrm{S}$, then the probability distance pdist of o in $\mathrm{s}$, satisfy the condition that $\forall \mathrm{s} \in \mathrm{S}$ : [d (o,s) $\leqq$ pdist $(\mathrm{o}, \mathrm{S})] \geq \varphi$.

Intuitively, pdist $(\mathrm{o}, \mathrm{S})$ is the probability of the data objects' number contained in $S$, which is in the spherical with the center of $o$ and the radius of pdist. Pdist $(o, S)$ can be indirectly used to estimate the density of $\mathrm{s}$, that is, the density of $\mathrm{S}$ is defined as the formula (1):

$$
\operatorname{pdens}(\mathrm{o}, \mathrm{S})=\frac{1}{\operatorname{pdist}(\mathrm{o}, \mathrm{S})}
$$

Suppose o be the center of $s, s \in S$, and the set of distance between $\mathrm{s}$ and $\mathrm{o}$ approximately follows the half Gaussian distribution, which is similar to the standard deviation. The standard distance of $\mathrm{s}$ and $\mathrm{o}$ is computed as the formula (2):

$$
\sigma(0, S)=\sqrt{\frac{\sum_{s \in S} d(o, s)^{2}}{|S|}}
$$


In Formula (2), because the distance $d(o, o)=0$ replaces the average $\mathrm{E}[\mathrm{d}(\mathrm{o}, \mathrm{S})]$, the standard distance of $\mathrm{o}$ and its standard deviation Stddev $(\mathrm{d}(\mathrm{o}, \mathrm{S}))$ is different. Therefore, we cannot assume that the set of distance values between $\mathrm{s}(\mathrm{s} \in \mathrm{S})$ and $\mathrm{o}$ follows normal distribution, but suppose that $S$ follow normal distribution with the centre o. So, we determine the correlation dataset $\mathrm{S}$ by taking advantage of this hypothesis, namely $\mathrm{S}=0$, and $\mathrm{S}$ is the nearest neighbors of $\mathrm{K}$. The distance between $\mathrm{o}$ and $\lambda$ corresponding probability set of $\mathrm{S}$ is defined as the formula (3):

$$
\operatorname{pdist}(\lambda, \mathrm{o}, \mathrm{S})=\lambda \cdot \sigma(o, S)
$$

Where, the parameter $\lambda$ is used for controlling the approximate value of the density.

In Formula (3), pdist $(\lambda, o, S)$ is used to estimate the density of the correlation set $\mathrm{s}$. For arbitrary data object $\mathrm{o}$, and $\mathrm{o} \in \mathrm{DS}$, suppose $\mathrm{S}(\mathrm{S} \subseteq \mathrm{DS})$ be the o's correlation dataset, then the related probability local outlier factor (PLOF) of $\lambda$ is defined as formula (4):

$$
\operatorname{PLOF} \lambda, \quad S(o)=\frac{p \operatorname{dist}(\lambda, o, S(o))}{E_{s \in S(o)}[\operatorname{pdist}(\lambda, s, S(s)]}-1
$$

Where E denotes the mean value, and S(o) denotes o's correlation dataset.

By using Gaussian error function, the probability value that the data objects $\mathrm{o}(\mathrm{o} \in \mathrm{DS})$ is outlier(local outlier probability (LoOP)) is defined as formula (5)

$$
\operatorname{LoOP}_{S}(\mathrm{o})=\max \left\{0, \operatorname{erf}\left(\frac{P L O F_{\lambda, S}(o)}{\sqrt{2} \cdot n P L O F}\right)\right\}
$$

Where $\mathrm{nPLOF}=\lambda \cdot \mathrm{E}\left[(\mathrm{PLOF})^{2}\right]$.

Remark. The value of $\lambda$ does not affect the sort of the probability values but only the outlier's probability values.

\section{RELEVANT SUBSPACE, SUBSPACE- DEPENDENT VECTOR, AND CONTEXTUAL OUTLIERS}

\subsection{Relevant Subspace}

Since the real datasets often contain the cluster (Cluster), which is generated from a variety of different mechanisms, different mechanisms may act on the different subsets of attribute dimensions[13]. So, the subset of attribute dimensions reflects that the appropriate mechanism can generate the corresponding subsets of data. Furthermore, it embodies a kind of similar local distribution characteristics, namely for the data subset generated from the specific mechanism, the subset of attribute dimensions has the characteristic of partial correlation: the common characteristic of local sparse distribution, namely some attribute dimensions are non-uniform distribution, while others are uniform distribution. Generally, only does the data subset generated from the specific mechanism reach a certain number, the common characteristics of local distributions can become apparent. Therefore, outlier can be regarded as the data objects which are not generated from such mechanism, namely, outlier is one type of data objects which are clearly inconsistent with the partial correlativity. For arbitrary data objects obj, attribute dimensions which are inconsistent with the common characteristics of the local sparse distribution features, can embody a kind of valuable information. They can also be described by a non-uniform distribution shown from the value of the local data attribute dimensions. However attribute dimensions, consistent with the common characteristics of the local sparse distribution features, cannot reflect any valuable information, but can be depicted by an uniform distribution on the value of the local data attribute dimensions. Referring to [14], the relevant subspace is redefined as follows:

Definition 1. For the detection of outlier, suppose DS be the ddimensions dataset, the global space $\mathrm{FS}, \mathrm{FS}=\mathrm{A}$ (the attribute set $\mathrm{A}$, and $A=\left\{A_{1}, A_{2}, \ldots, A_{d}\right\}$, and the nearest neighbors of data object obj are $\mathrm{N}$ (obj, FS) (namely the local dataset LDS). If the value of $\mathrm{N}(\mathrm{obj}, \mathrm{FS})$ conforms to the uniform distribution in the $\mathrm{A}_{\mathrm{i}}$ 's attribute dimension, then $\mathrm{A}_{\mathrm{i}}$ does not provide any valuable information and belongs to the attribute dimensions in the irrelevant subspace. Conversely, if the value of $\mathrm{N}(\mathrm{obj}, \mathrm{FS})$ conforms to the non-uniform distribution in $\mathrm{A}_{\mathrm{i}}$ attribute dimensions, then $A_{i}$ can provide some valuable information and belong to the attribute dimensions in the relevant subspace.

In [14], whether each subspace $S$ is a relevant subspace, is determined by using the nearest-neighbor $\mathrm{N}(\mathrm{o}, \mathrm{S})$, so for the datasets DS, the distribution characteristics of containing datasets in $\mathrm{S}$ will also change with the adjustment of $\mathrm{S}$ ' dimension. Therefore, if $\mathrm{S}$ is not FS, then $\mathrm{N}(\mathrm{o}, \mathrm{S})$ will lose the meaning of local dataset, and cannot effectively reflect the common features of the DS's local distribution characteristic in FS. However, according to definition 1, the relevant subspace is determined only by using N(obj, FS) in FS, and the above deficiencies in [14] can be effectively avoided.

\subsection{Subspace-dependent Vector}

In the local dataset LDS, since the value of uniform distribution attribute dimensions is more uniform, the sparsity (density) are similar or small differences, the uniform distribution attributes can be measured by taking the sparsity of the local attribute dimensions. Thus, the relevant subspace of data objects can be effectively identified by removing attribute dimensions of the uniform distribution and retaining non-uniform distribution attribute dimensions.

Suppose obj be the i-th data object in datasets DS, and LDS(obj) be the local dataset constructed from obj with K-NN methods. For LDS(obj), the sparsity (density) of obj in each attribute dimension can be measured by the following local sparse factor $\lambda_{\mathrm{ij}}$ :

$$
\lambda_{\mathrm{ij}}=\frac{\sum_{y \in p\left(x_{i j}\right)}\left(y-x_{i j}\right)^{2}}{K+1}
$$


Where $p\left(x_{i j}\right)$ is a sequence which is composed of LDS(obj)'s values on the attribute dimensions $A_{j}$, and $K$ is the number of nearest neighbors.

From formula (6), we can know that the local sparse factor $\lambda_{\mathrm{ij}}$ is the mean square value of Euclidean distance from each attribute dimension values of $A_{j}$ in the local dataset $\operatorname{LDS}(\mathrm{obj})$ and $\mathrm{x}_{\mathrm{ij}}$. Similar to formula (2), the larger the $\lambda_{\mathrm{ij}}$ is, and LDS(obj) on the attribute dimensions $A_{j}$ is more sparse. Conversely, the smaller the $\lambda_{i j}$ is, the more dense of LDS(obj) on the attribute dimensions $A_{j}$ is. In short, $\lambda_{\mathrm{ij}}$ can reflect the local sparsity (density) of local dataset LDS on its attribute dimension $A_{j}$

Obviously, from formula (6), we can determine the local sparse factor of each attribute dimension's of all the data objects, and accordingly generate the sparsity factor matrix $\left[Z_{\lambda}\right]_{n \times d}$ of the datasets DS. Furthermore, $\left[Z_{\lambda}\right]_{n \times d}=\left[\lambda_{i j}\right]$, where $n=|D S|, d$ is the degree of DS's dimension. From $\left[Z_{\lambda}\right]_{n \times d}$, we can construct the obj's local sparse factor matrix $\left[Z_{L \lambda}\right]_{k \times d}(o b j)$ on $\operatorname{LDS}(o b j)$, namely the local sparse factor matrix $\left[Z_{L \lambda}\right]_{k \times d}(o b j)$ of obj, where $\mathrm{k}=|\operatorname{LDS}(\mathrm{obj})|$.

To describe the difference of the attribute dimensions' local sparsity, we take the following local sparse difference factor $d_{i j}$ to depict the sparsity difference of the obj's $\mathrm{j}$-th attribute dimensions on LDS(obj).

$$
d_{i j}=\sqrt{\left|\frac{\left(\lambda_{i j}-C_{\lambda i j}\right)}{C_{\lambda i j}}\right|}
$$

Where: $C_{\lambda i j}$ is the mean square of $\left[Z_{L \lambda}\right]_{k \times d}(o b j)$ ' values on the attribute dimensions $\mathrm{A}_{\mathrm{j}}$.

From Formula (7), $d_{i j}$ depicts the difference degree of the local sparse factor $\lambda$ relative to the mean values. If $d_{i j}$ is greater, then the local sparse factor differences on the $j$-dimensional of the data objects obj's local sparse factor matrix $\left[\mathrm{Z}_{\mathrm{L} \lambda}\right]_{\mathrm{kxd}}(\mathrm{obj})$ is larger, namely, if the local density value of the obj's local dataset LDS on the attribute dimensions $A_{j}$ is more uneven, then the value of obj's local dataset LDS on the attribute dimensions is not uniform. Conversely, If $\mathrm{d}_{\mathrm{ij}}$ is less, then the local sparse factor differences on the j-dimensional of the data objects obj' $s$ local sparse factor matrix $\left[Z_{L \lambda}\right]_{k \times d}(o b j)$ is smaller, namely, if the local density value of the obj's local dataset LDS on the attribute dimensions $A_{j}$ is more even, then the value of obj's local dataset LDS on the attribute dimensions is more uniform. In conclusion, whether the value of obj's local dataset LDS on the attribute dimensions is uniform can be measured by using of $d_{i j}$.

Definition 2. Let $\varepsilon$ be the threshold of local sparse difference factor, $d_{i j}$ be the local sparse difference factor of the i-th data objects obj's $j$-th dimension. If $\mathrm{d}_{\mathrm{ij}}<_{\varepsilon}$, then $\mathrm{v}_{\mathrm{ij}}=0$, or else, $\mathrm{v}_{\mathrm{ij}}=1$, then $v_{i}=\left\{v_{i j}\right\}$, and $v_{i}$ is a vector defined by the obj's subspace, among which $i=1,2, \ldots|D S|, j=1,2, \ldots, d$, and $d$ is the dimension of DS. In $v_{i}$, the subspace constructed from the attribute dimensions with $\mathrm{v}_{\mathrm{ij}}$ value being 1 is called as the obj's relevant subspace, and the subspace constructed from the attribute dimensions with $v_{i j}$ value being 0 is called as the obj's irrelevant subspace.

When the dataset' dimension increases, the distance difference is not obvious, it will have no meaning by using distance to measure differences among data objects, but be meaningful for the nearestneighbor sort [24]. From formula(7) and definition 2, we can know that in LDS (obj), the local sparse factor of each neighbor loc_obj(loc_obj $\in$ LDS (obj) ) is obtained from LDS (loc_obj), that is, the vector defined by a data object obj's subspace is determined from the attribute dimensions of LDS (obj) and LDSloc_obj $\in$ LDS(obj)(loc_obj). Therefore, this subspace vector can accurately reflect the distribution characteristic of the local data, and ensure the correctness of the vector defined by the subspace. In addition, since the value of each dimension in the vector defined by the subspace is determined from the distribution condition of their corresponding attribute values, the determination of subspace vector has nothing to do with the dimension of DS.

In [13], the relevant subspace which is obtained from the correlated Pearson or Spearman methods is linear, and this method reflects the linear distribution characteristics of the local dataset. But by using the relevant subspace, we only discover that the outlier is obviously deviate from the linear distribution. The relevant subspace defined by definition 2 is determined from the local sparse difference factor of the local dataset, and constructed from the attribute dimensions whose density differences are more obvious. Similar to the analysis of [16], the vector defined by the subspace can determine arbitrarily nonlinear subspace, and be more accurate to describe the data objects with sparse density.

\subsection{Contextual Outlier}

From the above definition 2, if the relevant subspace does not exist in the i-th object obj, namely each dimension of the obj's subspace vector $\mathrm{v}$ is 0 , then it indicates that obj is consistent with local distribution features. If the relevant subspace exists in the i-th object obj, then it indicates that obj is inconsistent with the local distribution characteristics. In order to describe the degree of inconsistency (outlier), referring to [23], the outlier degree of obj (Factor (obj)) in the subspace is redefined as formula (10) by combining with definition 2 .

Let $v_{i}$ be a vector defined from the obj's subspace, $d$ be the $v_{i}$ 's dimensions, and $\lambda_{\mathrm{ij}}$ be the corresponding local sparse factor of $\mathrm{j}$ 's attribute dimensions (see: Formula(6)), $\mathrm{d}_{\mathrm{RS}}(\mathrm{o}, \mathrm{s})$ represents the Euclidean distance of data objects $s$ and $o$ in the relevant subspace RS. Referring to Formula (2) to (5), relative to the local dataset LDS (obj), the standard distance of obj and its relevant subspace RS is defined as the formula (8):

$$
\sigma_{R S}(o b j, L D S(o b j))=\sqrt{\frac{\sum_{o \in L D S(o b j)} d_{R S}(o b j, o)^{2}}{|L D S(o b j)|}}=\sqrt{\left(\sum_{j=1}^{d} v_{i} \cdot \lambda_{i j}\right)}
$$

In the obj's relevant subspace RS, loc_obj is the obj's arbitrary nearest neighbor, and the standard distance of loc_obj (loc_obj $\in \operatorname{LDS}($ obj $)$ )(let loc_obj be the $\mathrm{m}$-th data objects) relative to LDS(loc_obj) is defined as the formula (9).

$$
\begin{aligned}
& \left.\sigma_{\mathrm{RS}}\left(\mathrm{loc} \_ \text {obj,LDS(loc_obj }\right)\right)= \\
& \sqrt{\frac{\sum_{o \in L D S\left(l o c_{-} o b j\right)} d_{R S}\left(l o c \_o b j, o\right)^{2}}{\left|L D S\left(l o c \_o b j\right)\right|}}=\sqrt{\left(\sum_{j=1}^{d} v_{i} \cdot \lambda_{m j}\right)}
\end{aligned}
$$


According to formula (4), the probabilistic local outlier factor PLOF of obj in its relevant subspace RS is defined as the formula(10).

$$
P L O F_{R S}(o b j)=\frac{\sigma_{R S}(o b j, L D S(o b j))}{E_{o \in L D S(o b j)}\left(\sigma_{R S}(\mathrm{o})\right)}-1
$$

In the relevant subspace RS of obj, the probabilistic local outlier factor PLOF of loc_obj (loc_obj $\in \operatorname{LDS}(o b j)$ ) is defined as the fomula (11).

$$
P L O F_{R S}(\text { loc_obj })=\frac{\sigma_{R S}(\text { loc_obj,LDS }(\text { loc_obj }))}{E_{\text {o } \in L D S(\text { loc_obj })}\left[\sigma_{R S}(\mathrm{o}, \operatorname{LDS}(\mathrm{o}))\right]}-1
$$

In formula(10) and (11), $\operatorname{PLOF}_{\mathrm{RS}}(\mathrm{obj})$ and $\mathrm{PLOF}_{\mathrm{RS}}\left(\mathrm{loc} \_o b j\right)$ are similar to the local outlier factor LOF in the obj's relevant subspace RS, in that they both depict the local outlier degree of loc_obj in the obj's relevant subspace RS. It can be easily seen that, if we do not adopt relevant subspace RS, the definition of $\operatorname{PLOF}_{\mathrm{RS}}(\mathrm{obj})$ and $\mathrm{PLOF}_{\mathrm{RS}}\left(\mathrm{loc}_{-}\right.$obj $)$is no different in the space of FS composed of all the dimensions in DS (that is, $\mathrm{RS}=\mathrm{FS}$ ).

PLOF is not a probability value, nor a standardized value. If the value of PLOF is less than 0 , obj can be described as the normal data object, or else, if the value of PLOF is greater than 0 , the larger value PLOF shows the higher outlier degree of obj. But it is not comparable by adopting PLOF to measure different distribution of datasets [23]. Since there exists different dimensions of relevant subspace in datasets DS, the data distribution characteristic of different relevant subspace is different Therefore, PLOF in the different relevant subspace is not comparable.

To convert PLOF value to be a probability value, all dimensions are assumed to be independent each other, furthermore, each dimension has impact on the PLOF value [23]. According to the central limit theorem, we can assume that the space composed of all the dimensions obeys the normal distribution of E[PLOF $\left.{ }^{2}\right]$ (the variance with the center of 0)[23]. According to definition 2, if the value is less than $\varepsilon$, it is unable to provide valuable information, namely, attribute dimensions in the relevant subspace determined by definition 2 can have some impact on PLOF. Therefore, it can be assumed that $\mathrm{PLOF}_{\mathrm{o} \in \mathrm{LDS}(\mathrm{obj})}(\mathrm{o})$ in the relevant subspace (obj) (o) obeys the normal distribution of $\mathrm{E}_{\mathrm{o}} \in \in_{\mathrm{LDS}(\mathrm{obj})}\left[\left(\mathrm{PLOF}_{\mathrm{RS}}(\mathrm{o})\right)^{2}\right]$ (the variance with the center of 0 ), and also represents the probability of data objects being outlier with Gaussian error function. Referring to the Formula (5), the outlier degree(outlier factor) of obj in its relevant subspace RS is redefined as follows:

$$
\begin{aligned}
& \operatorname{Factor}(\mathrm{obj})= \\
& \max \left\{0, e r f\left(\frac{P L O F_{R S}(o b j)}{\left.\sqrt{2} \cdot \sqrt{E_{\mathrm{o} \in L D S(o b j) \cup o b j}\left[\left(P L O F_{R S}(\mathrm{o})\right)^{2}\right.}\right]}\right)\right\}
\end{aligned}
$$

Similar to [23], erf denotes the Gaussian error function. Because the parameter $\lambda$ in [23] does not affect the sort of outlier degree, but essentially divides the variable value of Gaussian error function to $\lambda$. Therefore we do not consider the selection of erf's value, similar to the condition of $\lambda$ being 1 in [23], if necessary, we can deal with in similar.

From Formula (10), (11) and (12), PLOF $_{\mathrm{RS}}$ (loc_obj) is not determined from the relevant subspace of loc_obj, but from the relevant subspace RS of obj since the inconsistency degree of Obj and its local distribution characteristics can be only embodied in the obj's relevant subspace RS when to determine Factor(obj).

In [23], each dimension is supposed to have the same effect on measuring outliers and calculating the data objects' PLOF in the space which is composed of all the dimensions. However, the properties of the uniform distribution which is also involved in calculating PLOF and many uniform distribution of attribute dimensions, are likely to make the PLOF values in formula (10) and (11) close to 0 . In short, attribute dimensions in the uniform distribution will not provide any valuable information for distinguishing between normal data and outliers, instead, their existence can probably change the original outlier into a normal data. In Formula (12), the outlier degree of data objects is determined in the relevant subspace RS. Since RS contains only attribute dimensions which follow the non-uniform distribution, and effectively avoids attribute dimensions in the uniform distribution to participating the calculation process of outlier degree. Meanwhile, formula (12) can be adapted to different distributions of data characteristics, as well as a variety of subspaces, namely it can be regarded as the unified measurement pattern in determining the outlier in the relevant subspace, and effectively improving the incomparable problem of measurement pattern in multiple subspaces in [19]. Especially, the normalization of PLOF is from the view of data sets in [23], and the PLOF in all over the datasets is supposed to obey the normal distribution. But, it is from a general perspective in formula (12), and the normalization of different clusters in datasets is also considered, furthermore, it was assumed that PLOF of the local dataset in the relevant subspace follows the normal distribution.

Definition 3 Let $v_{i}=\left\{v_{i j}\right\}$ be a vector defined from the subspace of data objects obj, where $i=1,2, \ldots,|D S|, j=1,2, \ldots, d$, and $d$ is the DS's dimension degree. In $\mathrm{v}_{\mathrm{i}}$, the corresponding Factor and attribute dimensions (obj) of the $\mathrm{v}_{\mathrm{ij}}$ value being 1 is called as the obj's contextual information, and the outlier with contextual information is called as contextual outlier.

From definition 3, we can know that the contextual information describes and depicts the attribute dimension information of the outlier's non-uniform distribution. Since the subspace of nonuniform distribution can effectively embody the outlier's valuable information, this type of information can effectively explain the characteristic, implication and the cause of outlier, and contribute to the understanding of outlier.

\section{THE RELEVANT SUBSPACE-BASED CONTEXTUAL OUTLIER MINING ALGORITHM}

The basic idea of outliers mining in the high-dimensional datasets DS is described as follows. Firstly, the global sparsity factor matrix is generated, and the obj's local sparse factor matrix which LDS corresponds to is constructed according to formula (6). Then, the local sparse difference factor which each attribute dimensions 
in obj corresponds to is computed by formula(7), and the obj's subspace vector is determined from definition 2 because the subspace vector can embody that obj is a relevant subspace or irrelevant subspace. If the relevant subspace exists in obj, then the obj's subspace is computed according to formula (12), otherwise, it shows that obj is a normal data object, and the outlier degree of obj is assigned with 0 . Finally, some data objects with the greatest outlier degree are selected as the outlier. The mainly algorithms are described as follows.

Algorithm COAS(Contextual Outlier Detection in Arbitrarily relevant subspaces)

Input: datasets DS, the sparsity threshold $\varepsilon$

Output: NN number of contextual outlier

$/ / *$ the default value $\varepsilon$ is 0.1

1) $\mathrm{L}=\mathrm{DS} ; / / * \mathrm{DS}$ is a dataset and is stored to $\mathrm{L}$

2) d=DS's dimension number;

$/ / *$ the number of DS's attributes

3) $n=|D S|$; //*the number of data objects in DS

4) $\mathrm{K}=\sqrt{n}$;

$/ / *$ the number of neighborhood is generally set with $\sqrt{n}$

5) $\operatorname{For}(i=1 ; i<=n ; i++)\{$

6) $\mathrm{L}[\mathrm{i}]=\mathrm{L} / /{ }^{*} \mathrm{~L}$ is the $\mathrm{i}$-th data object;

7) $\mathrm{L}[\mathrm{i}] . \mathrm{KNN}=\mathrm{ComputerKNN}(\mathrm{L}[\mathrm{i}], \mathrm{L}, \mathrm{K})$;

$/ / *$ referring to [13], compute the i-th data object' s KNN

8) $\mathrm{L}[\mathrm{i}] . \mathrm{LDS}=\mathrm{LKNN} \cup \mathrm{L}[\mathrm{i}]$;

$/ / *$ determine the i-th data object's local dataset

9) $\mathrm{L}[\mathrm{i}] . \lambda=$ ComputerL $\lambda(\mathrm{L}[\mathrm{i}] . \mathrm{LDS}, \mathrm{K})$;

// *according to definition 2, compute each attribute's sparsity factor of the i-th data object(see function 1 )

10) L. $\lambda$ Matrix.add(L[i]. $\lambda)$;

$/ / *$ the sparsity factor of the $i$-th data object was added to the line of sparsity factor matrix which the datasets DS is corresponding to.

11) \}

12) For $(i=1 ; i<=n ; i++)\{$

13) $\mathrm{L}[\mathrm{i}] . L D S \lambda$ Matrix $=$ Compute $\mathrm{L} \lambda \operatorname{Matrix}(\mathrm{L} . \lambda$ Matrix, L[i].LDS);

$/ / *$ select the i-data object's local sparse factor matrix according to the DS's sparsity factor matrix.

14) Factor $(L[i])=$ Computer Factor(L[i].LDS $\lambda$ Matrix,d,K);

$/ / *$ compute the outlier degree of the i-th data object(see function 2).

15) If (Factor(L[i])>0) \{OutlierMap.add(Factor, L[i]);

$/ / *$ save the value of the i-th data object's outlier degree

16) \}

17) Sort OutlierMap by Factor;

$/ / *$ the data object is sorted from big to small according to the outlier degree of the data objects.

18) Print Top(NN);

$/ / *$ Select NN number of data objects with the greatest outlier degree, and their corresponding contextual information(attribute dimensions which construct the relevant subspace) as contextual outlier.

\section{END COAS}

\section{Function1 ComputerL ${ }_{\lambda}(\mathrm{L}[\mathrm{i}] . \mathrm{LDS}, \mathrm{K})\{$} data

$/ / *$ compute each attribute's sparsity factor of the i-th object

1) For $(\mathrm{m}=1 ; \mathrm{m}<=\mathrm{d} ; \mathrm{m}++)\{$

2) $\operatorname{For}(j=1 ; i<=K+1 ; j++)\{$

3) $\mathrm{mSet}[\mathrm{j}]=(\mathrm{L}[\mathrm{i}] . \operatorname{LDS}[\mathrm{j}][\mathrm{m}])$;

$/ / *$ Add each attribute values of the L[i]'s local data in the m-th column to an array

4) \}

5) $\lambda_{\text {im }}=$ computer $\lambda_{\text {im }}($ Set $)$;

$/ / *$ according to formula (6), we compute the sparse factor of the attribute in the L[i]'s $m$-th dimension.

6) $\lambda_{\mathrm{i}}[\mathrm{m}]=\lambda_{\text {im }}$;

$/ / *$ Store the sparsity factor of the attribute in the L[i]'s $\mathrm{m}$-th dimension to an array.

7) \}

8) Return $\lambda_{\mathrm{i}}$;

END ComputerL ${ }_{\lambda}$

Function2 ComputerFactor(L[i].LDS $\lambda$ Matrix, d, K)

$/ / *$ compute the outlier degree of the i-th data object.

1) For $(j=1 ; j<=d ; j++)\{$

2) $x=0$;

3) For $(\mathrm{m}=1 ; \mathrm{m}<=\mathrm{K}+1 ; \mathrm{m}++)\{$

4) $\mathrm{x}+=\mathrm{L}[\mathrm{i}] . \operatorname{LDS} \lambda[\mathrm{m}][\mathrm{j}]$;

5) $\}$

6) Compute $\mathrm{d}_{\mathrm{ij}}(\mathrm{L}[\mathrm{i}])$ according to formula(7);

$/ / *$ the local sparse difference factor of the i-th data object's j-th column attribute

7) If $\left(\mathrm{d}_{\mathrm{ij}}(\mathrm{L}[\mathrm{i}])<\varepsilon\right)\{\mathrm{vi}[\mathrm{j}]=0 ;\}$ else $\left\{\mathrm{v}_{\mathrm{i}}[\mathrm{j}]=1 ;\right\}$

8) $\}$

9) If $\left(\left\|v_{i}\right\| 1=0\right)\{$ Factor(L[i] $\left.)=0 ;\right\}$ else $\{$ Factor(L[i]); $\}$

$/ / *$ computing the outlier degree of the i-th data objects according to formula (10).

10) Return Factor (L[i]);

END ComputerFactor

In COAS algorithm, the data object obj's subspace vector is determined through the local sparse difference factor, and the outlier degree is determined in the relevant subspace. If there exists irrelevant subspace, namely the subspace vector is total 0 , this indicates that the difference of the local density is close to 0 , obj is a normal data, and the outlier degree is assigned with 0 . The 
calculating of the data objects' $\mathrm{KNN}$ is realized by sequentially traverse, and the time complexity is o (n.n), but the average time complexity can be reduced o (n.logn) with the aid of the index structure[13]. For each local data object, each attribute's local sparse factor is calculated, the sparsity factor matrix of the original data is also generated, and the time complexity is o(n.k.d). In addition, the time complexity of determining each data object's subspace vector and outlier degree is $o(n \cdot k \cdot d)$. Therefore, the complexity of COAS algorithm is $o(n \cdot \log n+n \cdot k \cdot d+n \cdot k \cdot d)$.

\section{EXPERIMENTAL ANALYSIS}

We use java to implement the COAS and HiCS algorithms [16], COPalgorithm[13] algorithm and OUTRES algorithm[14], and conduct experiments by using a server that contains a Pentium D925 CPU, 256M memory, Windows XP operating system, ORACLE 9i DBMS development platform.

\subsection{Accuracy Analysis of The Algorithm}

To be convenient for outlier mining, 4 datasets from UCI are preprocessed according to the methods in $[3,14]$. As can be seen from table 1, "the outlier point category and its number" represents the type of outlier points and the retained data objects' number, and "the normal point category and its number" represents the type of normal points and the retained data objects' number.

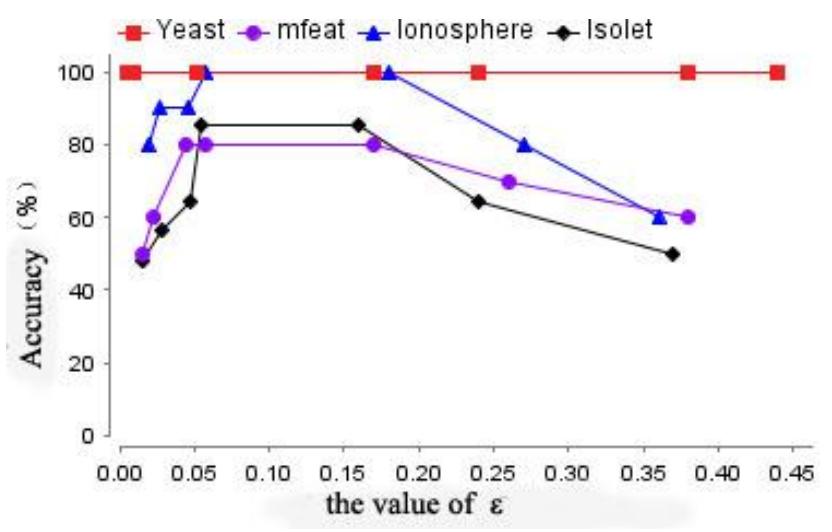

Fig.1 impact of $\varepsilon$ on the mining accuracy

Table 1. Preprocessing of UCI data

\begin{tabular}{|c|c|c|c|c|c|}
\hline $\begin{array}{c}\text { UCI data } \\
\text { sets }\end{array}$ & $\begin{array}{c}\text { number of Data } \\
\text { dimension }\end{array}$ & $\begin{array}{c}\text { normal point category } \\
\text { and its number }\end{array}$ & $\begin{array}{c}\text { outlier point category } \\
\text { and its number }\end{array}$ & $\begin{array}{c}\text { total number } \\
\text { of data }\end{array}$ & $\begin{array}{c}\text { number of } \\
\text { outlier }\end{array}$ \\
\hline Yeast & 8 & $\begin{array}{c}\text { ME3 400 CYT 400 MIT } \\
\text { 200 NUC 100 }\end{array}$ & ERL 5 & 1105 & 5 \\
\hline Ionosphere & 34 & Good 200 & Bad 5 & 235 & 5 \\
\hline mfeat & 649 & $\begin{array}{c}\text { 0-7 category, 150 of } \\
\text { each category }\end{array}$ & $8-9,5$ of each category & 1210 & 10 \\
\hline Isolet & 617 & $\begin{array}{c}1-20 \text { category, 200 of } \\
\text { each category }\end{array}$ & $\begin{array}{c}21-26 \text { category, } 8 \text { of each } \\
\text { category }\end{array}$ & 4048 & 48 \\
\hline
\end{tabular}

It can be seen from Fig. 1, when the local sparse difference factor threshold value $\varepsilon$ is bigger or smaller, the mining efficiency is lower. The main reason is that if $\varepsilon$ is set with the smaller value, most attribute dimensions are considered to be with significant differences in density according to definition 2 , and should situate in the relevant subspace, and thus cause the relevant subspace dimension to be expanded. For the high dimensional datasets, it will make results severely affected by "the curse of dimensionality", and the accuracy being decreased. Moreover, the outlier in the subspace could be hidden. For the low-dimensional datasets, the influence of "curse of dimensionality" is smaller, but it still hides the outlier in subspace. If $\varepsilon$ is set with the bigger value, most attribute dimensions are considered that the density difference is not obvious, and should situate in the irrelevant subspace, and thus cause to determine the outlier with significant difference in density as the normal data, and the accuracy will decrease. Therefore, the $\varepsilon$ curve in Fig. 1 shows "protruding" shape for high-dimensional data, while presents "near linear" shape for low-dimensional data. By definition 2, the local sparse difference factor of each attribute dimensions in unrelated is close to 0 , and the difference was not obvious. For the high dimensional datasets, once the threshold $\varepsilon$ becomes slightly smaller, a large number of attribute dimensions in the irrelevant subspace could be divided into the relevant subspace, and increases the "the curse of dimensionality" influence on the results. Therefore, the $\varepsilon$ curve which high dimensional of datasets correspond to is steeper on the left. As the threshold $\varepsilon$ increases to a certain degree, the outlier's attribute dimensions in the relevant subspace could be probably divided into the irrelevant subspace, and the outliers with significant differences in density are regarded as the normal data. Due to the significant differences exist in the density of the outlier, namely the difference of each attribute dimension's local sparse difference factor in the relevant subspace is always larger, the greater change of $\varepsilon$ can divide the outlier's attribute dimension in the relevant subspace to the irrelevant subspace, and thus the outlier may be considered to be a normal data. Therefore the curve of the threshold value $\varepsilon$ is generally flatter in the right. When the outlier's density in the datasets exist significantly difference, only the threshold value $\varepsilon$ become to larger value, can the outlier be regarded as a normal data. The mining accuracy is higher if $\varepsilon$ is within the larger range. Conversely, when the outlier's density difference is not obvious, once the threshold value $\varepsilon$ become to a smaller value, the outlier can be regarded as a normal data. The mining accuracy is higher if $\varepsilon$ is within the smaller range. Since the influence of "the curse of dimensionality" in the Yeast and Ionosphere datasets is small, the corresponding $\varepsilon$ curve is relatively flatter on the left, and the outlier's density exists significantly difference, so the high accuracy of gentle transition 
phase is wider. In short, the threshold $\varepsilon$ has a certain impact on the mining accuracy.

As can be seen from table 2, the accuracy of COAS algorithm is higher than that of the COP algorithm. The main reason is that COP algorithm is based on the distribution pattern, and the relevant subspace is obtained from all over the attribute dimensions by maximum likelihood estimation, and the accuracy is greatly influenced by the data distribution and the curse of dimensionality. In COAS algorithm, the dimensional differences of various properties is used to determine the vectors of subspace, and the accuracy is less affected by the data distribution and the curse of dimensionality, so the accuracy of COAS algorithm is higher than that of the COP algorithm. Since the determination of relevant subspace is based on a global approach in HiCS algorithm, and the relevant subspace candidates are searched through the similar Apriori algorithm, it may lead to the relevant subspace discovered from the datasets is all incomplete, and some outlier cannot be found. But the COAS algorithm can find the relevant subspace in the data, and also effectively discover the outliers hidden in any relevant subspace. Similar to HiCS algorithm, the relevant subspace in OUTRES algorithm is also based on a global, and thus the accuracy of OUTRES algorithm is lower than COAS algorithm. In short, the accuracy of COAS is relatively higher on the different datasets, especially with pretty obvious advantages in the high-dimensional data.

Table 2. Accuracy comparisons on UCI data sets

\begin{tabular}{|c|c|c|c|c|c|c|c|c|c|}
\hline \multirow{2}{*}{$\begin{array}{l}\text { UCI data } \\
\text { sets }\end{array}$} & \multirow{2}{*}{$\begin{array}{l}\text { Number } \\
\text { of outlier }\end{array}$} & \multicolumn{2}{|c|}{ COP algorithm } & \multicolumn{2}{|c|}{$\begin{array}{c}\text { COAS algorithm } \\
(\varepsilon=0.1)\end{array}$} & \multicolumn{2}{|c|}{$\begin{array}{c}\text { HiCS algorithm } \\
(\alpha=0.1, M=50, \\
\text { candidate_cutoff }=50)\end{array}$} & \multicolumn{2}{|c|}{$\begin{array}{l}\text { OUTRES algorithm } \\
\quad(\varepsilon=15, \alpha=0.01)\end{array}$} \\
\hline & & Outlier & Accuracy & Outlier & Accuracy & Outlier & Accuracy & Outlier & Accuracy \\
\hline Yeast & 5 & 5 & $100 \%$ & 5 & $100 \%$ & 5 & $100 \%$ & 5 & $100 \%$ \\
\hline Ionosphere & 5 & 4 & $80 \%$ & 5 & $100 \%$ & 3 & $60 \%$ & 3 & $60 \%$ \\
\hline mfeat & 10 & 4 & $40 \%$ & 9 & $80 \%$ & 5 & $50 \%$ & 6 & $60 \%$ \\
\hline Isolet & 48 & 18 & $37.5 \%$ & 41 & $85.4 \%$ & 24 & $50 \%$ & 31 & $64.6 \%$ \\
\hline
\end{tabular}

\subsection{Efficiency Analysis of The Algorithm}

The Experimental datasets are discrete Star spectrum datasets SDSS provided by China National Solar Observatory [2, 18, 25], and the experimental results are shown as figures 2, 3 and 4 .

As can be seen from Fig. 2, the influence of the local sparse difference factor eis smaller on the mining efficiency of COAS algorithm. The main reason is that most operations of COAS algorithm focus on searching for the k-nearest neighbor, and it has nothing to do with $\varepsilon$. The value of $\varepsilon$ only affects the value of each dimension in the subspace vector. The subspace vector with totally 0 can be possibly increased with the increasing of $\varepsilon$. Due to it need not be computed according to formula (14) when the space calculation vector is totally 0 , so with the increasing of $\varepsilon$, the overall amount of computation can be reduced. In short, the value of $\varepsilon$ has less impact on the mining efficiency.

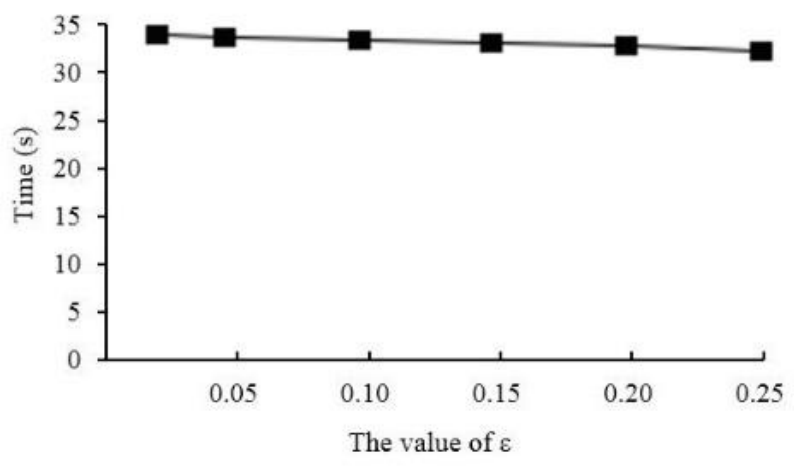

Fig. 2 Mining Efficiency On different $\varepsilon$ (Spectral data Records=10000, dimensions = 200) 


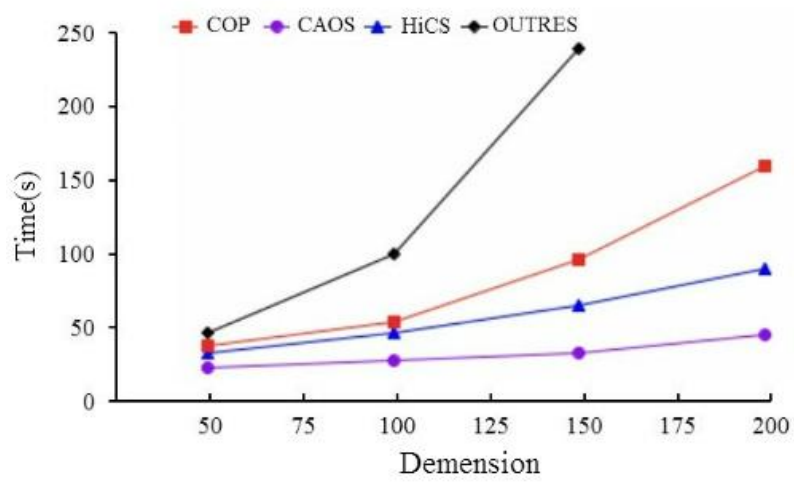

Fig. 3 Mining Efficiency On attribute dimension $($ spectral data Records=10000, $\varepsilon=0.1$ )

It can be seen from Fig. 3, when the number of data sets are the same, the pruning effects are very limited while the relative dimension of the subspace is higher because the complexity of COP algorithm is the cube of the dimension, and the complexity of OUTRES algorithm is exponential with the dimension in spite of the fact that the pruning strategies can be taken to reduce the time when determining the relative subspace in [18]. Similar to Apriori algorithm, two dimension subspaces of all the dimensions are first generated in HiCS algorithm, and the candidate set of two dimension subspace are selected, then three dimension candidate set are generated from the two dimension of candidate set, until $\mathrm{d}+1$ dimension subspace cannot be generated from the candidate set $\mathrm{d}+1$ dimension candidate subspace, in the end the candidate set of $\mathrm{d}$ dimension subspace is the related subspace set. The candidate_cutoff is retained in each candidate set (namely the maximum number of candidate set's items, and the suitable candidate item is more or less than 500 which is obtained from many times of experiments in [19]) . The pruning is constantly processed in the generation process in spite of that the exponent of dimension is effectively done with, the candidate set is acquired through the sorting of the difference value of subspaces. The difference value of each subspace is obtained through $\mathrm{M}$ iterations process, and the impact of the dimension degree is larger and the computation amount is also quite large. The computation complexity of the COAS Algorithm is relatively small since it is nearly linear relationship with the dimension. Thus, the efficiency of COAS algorithm is higher than that of COP algorithm OUTRES algorithm and HiCS algorithm.

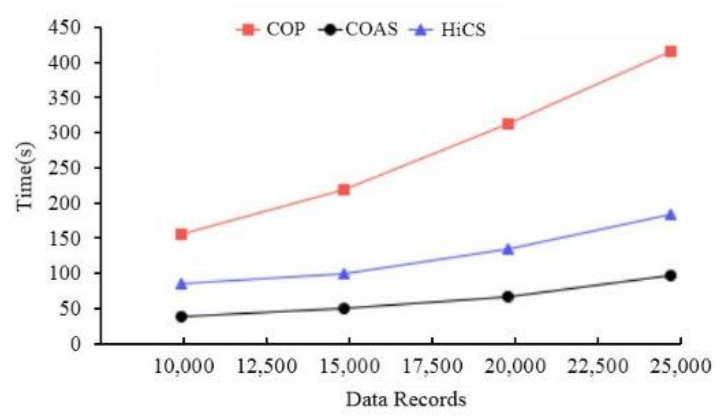

Fig. 4 Mining Efficiency On Different Data Sets(Data dimension=200, $\varepsilon=0.1$ )

As can be seen from Fig. 4, the time complexity of three algorithms 3 is nearly linear, but the complexity of COP algorithm is the cube of the dimension, while the complexity of COAS algorithm is nearly to the liner relationship with the dimension. Therefore, the tilt degree of COP algorithm is greater than COAS algorithm, and its efficiency is lower than COAS algorithm. Similar to the algorithm analysis of HiCS algorithm in Fig. 4, the impact of dimension is still high and the tilt degree of HiCS algorithm is greater than that of COAS algorithm and the efficiency is lower than the COAS algorithm although the Apriori approach and the pruning strategies are taken by it.

\subsection{Understandability of The Algorithm}

To evaluate the understandability of the algorithm, we made experiments on the Wholesale customers Data Set from UCI datasets (http://archive.ics.uci.edu/ml/datasets/Wholesale+ customers). The data set refers to clients of a wholesale distributor. It includes the annual spending in monetary units (m.u.) on diverse product categories in different sales channels and regions. This data set contains a total of 440 pieces of data, among which every piece of data denotes some information on this wholesale distributors, and is composed of 8 dimensions: CHANNEL, REGION, Fresh, Milk, Grocery, Frozen, Detergents_Paper and Delicatessen. In this analysis, we first make preprocess with the data. Since CHANNEL and REGION are qualitative data, these dimensions of data are ignored during the calculation process. In the case of local sparse difference factor thresholds $\varepsilon=0.1$, two outliers whose outlier factors are the largest, are obtained by COAS algorithm and shown in Table 3 . Table 4 and Table 5 are the two outliers' reference or local data set, respectively. 
Table 3, Outliers

\begin{tabular}{|c|c|c|}
\hline No. & Oulier factor & Relevant subspace \\
\hline obj183 & 0.886 & $\begin{array}{c}\{\text { Fresh, Milk, Frozen, } \\
\text { Detergents_Paper, Delicatessen }\}\end{array}$ \\
\hline obj86 & 0.886 & $\begin{array}{c}\{\text { Fresh, Milk, Frozen, } \\
\text { Detergents_Paper, Delicatessen }\}\end{array}$ \\
\hline
\end{tabular}

As can be seen from Table 3, Table 4 and Table 5, the outlier data obj183 of $\{36847,43950,20170,36534,239,47943\}$ contains the following context information: the reference data set of $\{183,23$, $325,427,435,87,196,202,265,384,71,24,12,92,40,47,103$, $165,45,251,254\}$, the relevant subspace \{Fresh, Milk, Frozen, Detergents_Paper, Delicatessen $\}$ and the outlier Factor $=0.886$. The outlier data obj86 of $\{22925,73498,32114,987,20070,903\}$ contains the following context information: the reference data set of $\{86,47,23,61,211,56,92,319,251,49,171,45,28,437,65$, 384, 265, 163, 331, 216, 309\}, the relevant subspace \{Fresh, Milk, Frozen, Detergents_Paper, Delicatessen $\}$ and the outlier Factor $=0.886$. The outlier data obj183 can be explained as follows: the annual spending on Fresh, Milk, Frozen, Detergents_Paper and Delicatessen are 36847, 43950, 36534, 239, and 47943 respectively. The difference between these data and the reference data set is very large, i.e. the values on Fresh, Milk, Frozen and Delicatessen are much larger, while the value on Detergents_Paper is much smaller. In addition, the outlier data obj86 can be explained as follows: the annual spending on Fresh, Milk, Frozen, Detergents_Paper, and Delicatessen are 22925, 73498, 987, 20070, and 903 respectively. The difference bteween these data and the reference data set is very large, i.e. the values on Fresh, Milk and Detergents_Paper are much larger, while the values on Frozen and Delicatessen are much smaller.

Table 4. Reference or Local data set of outlier object 183

\begin{tabular}{|c|c|c|c|c|c|c|}
\hline No. & Fresh & Milk & Grocery & Frozen & Detergents_Paper & Delicatessen \\
\hline obj183 & 36847 & 43950 & 20170 & 36534 & 239 & 47943 \\
\hline obj12 & 31714 & 12319 & 11757 & 287 & 3881 & 2931 \\
\hline obj23 & 26373 & 36423 & 22019 & 5154 & 4337 & 16523 \\
\hline obj24 & 22647 & 9776 & 13792 & 2915 & 4482 & 5778 \\
\hline obj40 & 24025 & 4332 & 4757 & 9510 & 1145 & 5864 \\
\hline obj45 & 5181 & 22044 & 21531 & 1740 & 7353 & 4985 \\
\hline obj47 & 44466 & 54259 & 55571 & 7782 & 24171 & 6465 \\
\hline obj71 & 18291 & 1266 & 21042 & 5373 & 4173 & 14472 \\
\hline obj87 & 43265 & 5025 & 8117 & 6312 & 1579 & 14351 \\
\hline obj92 & 9198 & 27472 & 32034 & 3232 & 18906 & 5130 \\
\hline obj103 & 56082 & 3504 & 8906 & 18028 & 1480 & 2498 \\
\hline obj165 & 15615 & 12653 & 19858 & 4425 & 7108 & 2379 \\
\hline obj196 & 30624 & 7209 & 4897 & 18711 & 763 & 2876 \\
\hline obj202 & 25203 & 11487 & 9490 & 5065 & 284 & 6854 \\
\hline obj251 & 6134 & 23133 & 33586 & 6746 & 18594 & 5121 \\
\hline obj254 & 10379 & 17972 & 4748 & 4686 & 1547 & 3265 \\
\hline obj265 & 5909 & 23527 & 13699 & 10155 & 830 & 3636 \\
\hline obj325 & 32717 & 16784 & 13626 & 60869 & 1272 & 5609 \\
\hline obj384 & 10683 & 21858 & 15400 & 3635 & 282 & 5120 \\
\hline obj427 & 31012 & 16687 & 5429 & 15082 & 439 & 1163 \\
\hline obj435 & 29703 & 12051 & 16027 & 13135 & 182 & 2204 \\
\hline
\end{tabular}


Table 5. Reference or Local data set of outlier object 86

\begin{tabular}{|c|c|c|c|c|c|c|}
\hline No. & Fresh & Milk & Grocery & Frozen & Detergents_Paper & Delicatessen \\
\hline obj86 & $\mathbf{2 2 9 2 5}$ & $\mathbf{7 3 4 9 8}$ & 32114 & $\mathbf{9 8 7}$ & $\mathbf{2 0 0 7 0}$ & $\mathbf{9 0 3}$ \\
\hline obj23 & 26373 & 36423 & 22019 & 5154 & 4337 & 16523 \\
\hline obj28 & 4113 & 20484 & 25957 & 1158 & 8604 & 5206 \\
\hline obj45 & 5181 & 22044 & 21531 & 1740 & 7353 & 4985 \\
\hline obj47 & 44466 & 54259 & 55571 & 7782 & 24171 & 6465 \\
\hline obj49 & 4967 & 21412 & 28921 & 1798 & 13583 & 1163 \\
\hline obj56 & 4098 & 29892 & 26866 & 2616 & 17740 & 1340 \\
\hline obj61 & 35942 & 38369 & 59598 & 3254 & 26701 & 2017 \\
\hline obj65 & 85 & 20959 & 45828 & 36 & 24231 & 1423 \\
\hline obj92 & 9198 & 27472 & 32034 & 3232 & 18906 & 5130 \\
\hline obj163 & 5531 & 15726 & 26870 & 2367 & 13726 & 446 \\
\hline obj171 & 200 & 25862 & 19816 & 651 & 8773 & 6250 \\
\hline obj211 & 12119 & 28326 & 39694 & 4736 & 19410 & 2870 \\
\hline obj216 & 2532 & 16599 & 36486 & 179 & 13308 & 674 \\
\hline obj251 & 6134 & 23133 & 33586 & 6746 & 18594 & 5121 \\
\hline obj265 & 5909 & 23527 & 13699 & 10155 & 830 & 3636 \\
\hline obj309 & 918 & 20655 & 13567 & 1465 & 6846 & 806 \\
\hline obj319 & 9759 & 25071 & 17645 & 1128 & 12408 & 1625 \\
\hline obj331 & 11223 & 14881 & 26839 & 1234 & 9606 & 1102 \\
\hline obj384 & 10683 & 21858 & 15400 & 3635 & 282 & 5120 \\
\hline obj437 & 14531 & 15488 & 30243 & 437 & 14841 & 1867 \\
\hline
\end{tabular}

\section{CONCLUSION}

In the high-dimensional and massive datasets, the implantation of data mining task from the relevant subspace, constructed from the meaningful dimension, is one of the hot spots and difficult problems. In this paper, a relevant subspace based contextual outlier mining algorithm is proposed. In the algorithm, the relevant subspace is redefined by using the local sparse degree of attribute dimensions in the local data set, the attribute dimensions constituting the relevant subspace is regarded as the data objects' contextual information, and accordingly the influence of irrelevant attribute dimensions on the outlier can be effectively avoided and the context-sensitive outliers hidden in the subspace can be detected. Furthermore, the algorithm has the better mining effects. The future work would focus on the degree of outlier values are smaller when the local dataset are difficult to reflect the distribution characteristics of clusters.

\section{ACKNOWLEDGMENTS}

This work is partially supported by the National Natural Science Foundation of P. R. China (61572343). Xiao Qin's work is supported by the U.S. National Science Foundation under Grants CCF-0845257(CAREER).

\section{REFERENCES}

[1] Knox E M, Ng R T. Algorithms for mining distance-based outliers in large datasets[C].In Proceedings of the International Conference on Very Large Data Bases. 1998.
[2] Zhang J F, Zhang S L, Chang K H, and Qin X. An Outlier Mining Algorithm Based on Constrained Concept Lattice, International Journal of Systems Science, 2014,45(5):11701179.

[3] Pham N, Pagh R. A near-linear time approximation algorithm for angle-based outlier detection in high-dimensional data[C]. In Proceedings of the 18th ACM SIGKDD international conference on Knowledge discovery and data mining. ACM, 2012: 877-885.

[4] Sequeira K, Zaki M. ADMIT: anomaly-based data mining for intrusions[C]. In Proceedings of the eighth ACM SIGKDD international conference on Knowledge discovery and data mining. ACM, 2002: 386-395.

[5] Lazarevic A, Ertoz L, Kumar V, et al. A comparative study of anomaly detection schemes in network intrusion detection[J]. Proc. SIAM, 2003.

[6] Liu H, Shah S, Jiang W. On-line outlier detection and data cleaning[J]. Computers \& chemical engineering, 2004, 28(9): 1635-1647.

[7] Barnett V, Lewis T. Outliers in statistical data[M]. New York: Wiley, 1994.

[8] Ramaswamy S, Rastogi R, Shim K. Efficient algorithms for mining outliers from large data sets[C]. ACM SIGMOD Record. ACM, 2000, 29(2): 427-438. 
[9] Breunig M M, Kriegel H P, Ng R T, et al. LOF: identifying density-based local outliers[C]. ACM Sigmod Record. ACM, 2000, 29(2): 93-104.

[10] Papadimitriou S, Kitagawa H, Gibbons P B, et al. Loci: Fast outlier detection using the local correlation integral[C]. 19th International Conference on Data Engineering, 2003: 315-326.

[11] Sarawagi S, Agrawal R, Megiddo N. Discovery-driven exploration of OLAP data cubes[M]. Springer Berlin Heidelberg, 1998.

[12] Kriegel H P, Zimek A. Angle-based outlier detection in highdimensional data[C]. In Proceedings of the 14th ACM SIGKDD international conference on Knowledge discovery and data mining. ACM, 2008: 444-452.

[13] Kriegel H P, Kroger P, Schubert E, et al. Outlier detection in arbitrarily oriented subspaces[C]. 2012 IEEE 12th International Conference on Data Mining (ICDM), 2012: 379388.

[14] Muller E, Schiffer M, Seidl T. Statistical selection of relevant subspace projections for outlier ranking[C]. 2011 IEEE 27th International Conference on Data Engineering (ICDE), 2011: 434-445.

[15] Aggarwal C C, Yu P S. Outlier detection for high dimensional data[C]. ACM Sigmod Record. ACM, 2001, 30(2): 37-46.

[16] Keller F, Muller E, Bohm K. HiCS: high contrast subspaces for density-based outlier ranking[C]. 2012 IEEE 28th International Conference on Data Engineering (ICDE), 2012: 1037-1048.

[17] Aggarwal C C, Philip S Y. An effective and efficient algorithm for high-dimensional outlier detection[J]. The VLDB journal, 2005, 14(2): 211-221.
[18] Zhang J, Jiang Y, Chang K H, et al. A concept lattice based outlier mining method in low-dimensional subspaces[J]. Pattern Recognition Letters, 2009, 30(15): 1434-1439

[19] Kriegel H P, Kröger P, Schubert E, et al. Outlier detection in axis-parallel subspaces of high dimensional data[M]. Advances in Knowledge Discovery and Data Mining. Springer Berlin Heidelberg, 2009: 831-838.

[20] Bouguessa M, Wang S. Mining projected clusters in highdimensional spaces[J]. IEEE Transactions on Knowledge and Data Engineering, 2009, 21(4): 507-522.

[21] Tang G, Pei J,et al. Mining multidimensional contextual outliers from categorical relational data [C].In Proceedings of Scientific and Statistical Database Management'13, Baltimore, MD, USA July 29 - 31 2013:1-4

[22] Wang X, Davidson I. Discovering contexts and contextual outliers using random walks in graphs[C]. In Proceedings of Ninth IEEE International Conference on Data Mining, 2009: 1034-1039.

[23] Kriegel H P, Kröger P, Schubert E, et al. LoOP: local outlier probabilities[C]. In Proceedings of the 18th ACM conference on Information and knowledge management. ACM, 2009: 1649-1652.

[24] Houle M E, Kriegel H P, Kröger P, et al. Can shared-neighbor distances defeat the curse of dimensionality?[C]. In Proceedings of Scientific and Statistical Database Management'10, Springer Berlin Heidelberg, 2010, 6187: 482500.

[25] Zhang J, Zhao X, Zhang S, et al. Interrelation analysis of celestial spectra data using constrained frequent pattern trees[J]. Knowledge-Based Systems, 2013, 41: 77-88. 